\title{
An Atmospheric Radiation Measurement Value-Added Product to Retrieve Optically Thin Cloud Visible Optical Depth using Micropulse Lidar
}

October 2006

Chaomei Lo

Jennifer M. Comstock

Connor Flynn

Pacific Northwest National Laboratory

Richland, Washington

Work supported by the U.S. Department of Energy,

Office of Energy Research, Office of Biological and Environmental Research 


\section{Contents}

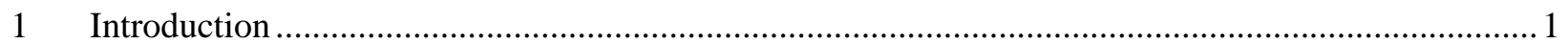

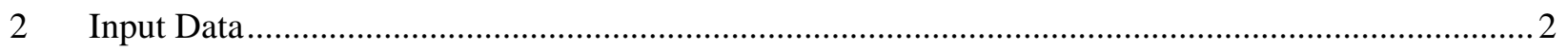

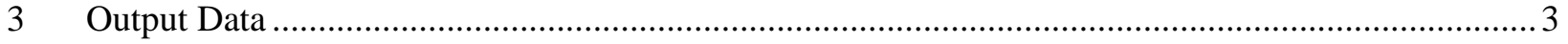

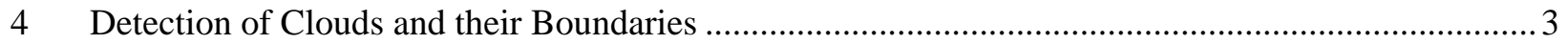

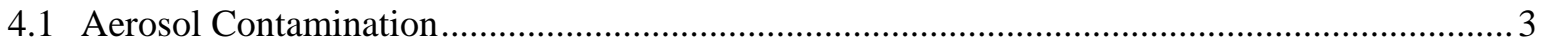

4.2 Attenuation of the Lidar Backscatter Signal....................................................................... 4

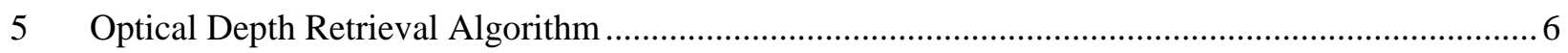

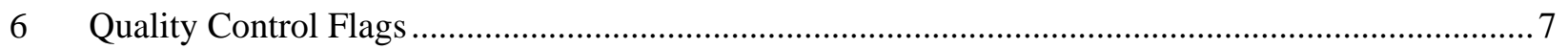

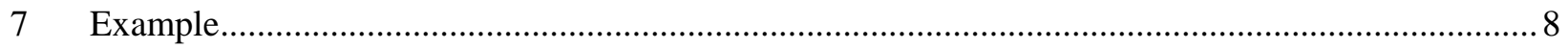

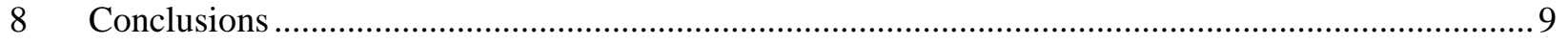

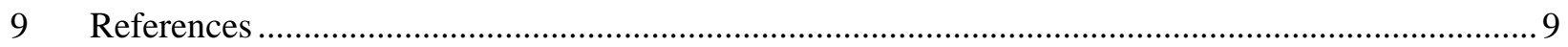

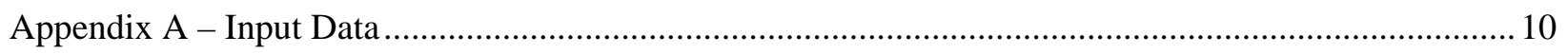

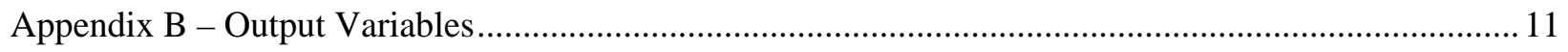

Figures

1 Top panel: Example of the aerosol contamination below the cloud ................................. 5

2 From top down: (1) Lidar backscatter intensities and calculated cloud boundaries (2) Retrieved optical depth (3) Backscatter-to-extinction ratio in $\mathrm{sr}^{-1}$ (4) QC bits................. 8

\section{Tables}

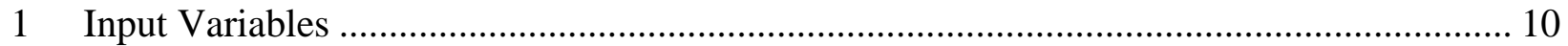

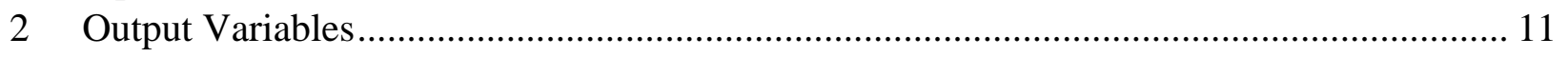




\section{Introduction}

The primary factor that regulates the radiative energy balance in the Earth's atmosphere is clouds. Optically thin clouds can act to either heat or cool the atmosphere depending on their temperatures and optical depth $(\tau)$. Optically thin clouds (e.g., $\tau<3$ ) include upper tropospheric ice clouds (i.e., cirrus), and low and middle level water or mixed phase clouds. Ice clouds are composed of ice crystals that are typically hexagonal in shape, but can also consist of irregular shapes such as aggregates and chains. Due to the difficulties of measuring the shape and scattering properties of ice crystals in clouds, modeling the scattering and absorption of radiation in the atmosphere is challenging. To improve the theoretical calculations of the climatic effects of clouds, there has been an increased interest in the retrieval of cloud macrophyscal, microphysical, and radiative properties.

The purpose of the Micropulse Lidar (MPL) Cloud Optical Depth (MPLCOD) ValueAdded Product (VAP) is to retrieve the visible (short-wave) cloud optical depth for optically thin clouds using MPL. The advantage of using the MPL to derive optical depth is that lidar is able to detect optically thin cloud layers that may not be detected by millimeter cloud radar or radiometric techniques. The disadvantage of using lidar to derive optical depth is that the lidar signal becomes attenuation limited when $\tau$ approaches 3 (this value can vary depending on instrument specifications). As a result, the lidar will not detect optically thin clouds if an optically thick cloud obstructs the lidar beam.

The MPLCOD algorithm uses data from the MPLNOR (normalized backscatter) VAP as input. The MPLNOR VAP applies overlap, range, dead-time, and afterpulse corrections to the lidar returned energy. We also use radiosonde (SONDE) profiles of temperature and pressure to compute the molecular backscatter coefficient. The radiosonde file is chosen for each lidar backscatter profile based on its closest time to that of the balloon launch time.

The retrieval algorithm is derived from Comstock et al. (2001), which retrieves visible optical depth and backscatter-to-extinction ratio $(k)$ at the lidar wavelength for each backscatter profile. The variable $k$ parameter allows for changes in the microphysical properties of the cloud with time. Both the input MPLNOR and SONDE data could have multiple files each day. The output netcdf file for the MPLCOD VAP combines the multiple input files to create a single output file for each day. 


\section{Input Data}

The input files for this VAP are standard ARM data files in NetCDF format. To run this VAP, we need the following input files and data ${ }^{1}$ :

\section{MPL NORmalization and Cloud Detection VAP:}

xxxmplnor1campFF.c1. YYYYMMDD.hhmmss - 1 min data generally, it may contain time gaps like March 6, 2000.

This data stream contains the following:

- Corrected and normalized backscatter (backscatter)

- "Sensitive” cloud mask (cloud_mask_1)

- “Robust” cloud mask (cloud_mask_2)

- Cloud top height

- Cloud base height

\section{Balloon-Borne Sounding System (SONDE):}

xxxsondewrpnFF.a1.YYYYMMDD.hhmmss - 2 second data

or

xxxsondewnpnFF.a1.YYYYMMDD.hhmmss - 2 second data

The variables used from this datastream are:

- Temperature profile

- $\quad$ Pressure profile

where:

Xxx - the locations of the instrument (nsa, sgp, twp)
FF - facility (e.g., C1)

${ }^{1}$ For details of the input variables see Appendix A. 


\section{Output Data}

The name of the output file is:

xxxmplcod1comflyFF.C1.YYYYMMDD.hhmmss - time sample resolution is the same as the mplnor vap input described above.

Where:

$$
\begin{aligned}
& \mathrm{xxx} \quad-\quad \text { the site of the instrument } \\
& \text { mpl - the main instrument name } \\
& \text { cod } \quad-\text { optical depth } \\
& \text { com - identifies that this is J Comstock's version } 1 \text { VAP } \\
& \text { fly _ - identifies the use of C Flynn's code to find the clear sky } \\
& \text { above and below the cloud etc. } \\
& \mathrm{FF} \quad-\text { facility } \\
& \text { YYYY - year, MM - month of the year, DD - day of the month, hh - hour of the } \\
& \text { day, mm - minute of the hour, ss - second of the minute of data start }
\end{aligned}
$$

The detailed variable description is in Table 2 of Appendix B.

\section{Detection of Clouds and their Boundaries}

An important element for the retrieval of optical depth using a single wavelength lidar is the identification of cloud and aerosol free regions above and below the cloud. It is also important to identify times when the lidar signal is attenuation limited. The MPL backscatter signal is carefully screened to identify regions of aerosol contamination or virga below the cloud and reliable molecular signal return above the cloud. The field "cloud_mask_2" data from MPLNOR are used to detect the existence of clouds. The top panel of Figure 1 demonstrates the aerosol contamination from a backscatter profile.

\subsection{Aerosol Contamination}

Below the cloud, aerosol contamination is identified by calculating the ratio of the measured backscatter coefficient ( $\beta$ ) divided by the computed attenuated molecular backscatter coefficient $\left(\beta_{\mathrm{m}}\right)$. The parameter $\beta_{\mathrm{m}}$ is related to the Rayleigh backscatter cross section $\sigma_{\mathrm{m}}=5.45 \mathrm{e}-28 *(550 / \lambda)^{4}$ where $\lambda$ is the lidar wavelength in $\mathrm{nm}$ and $\sigma_{\mathrm{m}}$ is in $\mathrm{cm}^{2} \mathrm{sr}^{-1}$ (Measures 1992). The molecular backscatter volume coefficient is then $\beta_{\mathrm{m}}=\mathrm{N}_{\mathrm{d}} \sigma_{\mathrm{m}}$ where the number density $\mathrm{N}_{\mathrm{d}}$ is derived using the ideal gas law. Note that for pure Rayleigh scattering, the extinction coefficient $\alpha_{\mathrm{m}}=(8 \pi / 3) \beta_{\mathrm{m}}$. Once $\beta_{\mathrm{m}}$ is computed, the profile is corrected for atmospheric attenuation using the equation:

$$
\beta_{m}^{\prime}(z)=\beta_{m}(z) \exp \left(-2 \int \alpha_{m}\left(z^{\prime}\right) d z^{\prime}\right) .
$$


Once the ratio $\beta / \beta_{\mathrm{m}}$ is computed, aerosol contamination is identified when the ratio is less than a specified threshold. In the MPLCOD VAP, the threshold is $5 \%$ of the mean ratio of the below cloud points.

\subsection{Attenuation of the Lidar Backscatter Signal}

To identify attenuation limited lidar profiles, the above cloud signal is compared with the attenuated molecular profile. The following list summarizes the checks performed to ensure that the lidar signal is not attenuated:

1. The measured backscatter signal should be greater than a threshold value which is the minimum of the positive backscatter.

2. We require that the ratio of measured backscatter to attenuated molecular signal above the cloud to be less than that below the cloud.

3. Progressively step through the lidar backscatter profile above the cloud using 11 range bins to determine that no cloud is detected in the backscatter signal. If the backscatter in the lowest above cloud range bin is within $5 \%$ of a numerical fit to the backscatter in the next 10 range bins, then the signal is determined to be "above cloud" and not "in cloud." Otherwise the lowest bin is discarded and an additional bin is added at the top. The analysis is repeated until the $5 \%$ criterion is met.

4. Once a cloud free interval is determined above cloud, we determine if the lidar signal is attenuation limited within this interval by comparing the backscatter signal with the attenuated $\beta_{\mathrm{m}}$ profile in this interval. Assuming the interval has NPTS, we compare the ratio of the measured backscatter profile $\beta(1: \mathrm{NPTS} / 2) / \beta(\mathrm{NPTS} / 2$ :NPTS) with the ratio of the attenuated molecular profile $\beta_{\mathrm{m}}(1: \mathrm{NPTS} / 2) / \beta_{\mathrm{m}}(\mathrm{NPTS} / 2: \mathrm{NPTS})$. If the measured ratio and the attenuated molecular ratio agree within $5 \%$, then we assume the signal is not attenuated. If they are not in agreement, then the upper one-third of the points are discarded. The analysis is repeated until it is determined that the backscatter is not attenuated or until there are no longer sufficient points in the above cloud interval to retrieve the optical depth.

In Figure 1, an example of a backscatter profile that is attenuation limited above the cloud (middle panel), an example of a profile that has aerosol and virga free regions both above and below the cloud, and suitable molecular return above the cloud (bottom panel) are demonstrated. Successful determination of cloud and aerosol free intervals below and above the cloud is critical to the optical depth retrieval. Other checks performed are to identify clouds that are too low (i.e., fog) and cases when significant molecular signal is unavailable above cloud. Once the appropriate intervals above and below the cloud are determined (i.e., Figure 1, bottom panel), the optical depth retrieval is performed. If the 

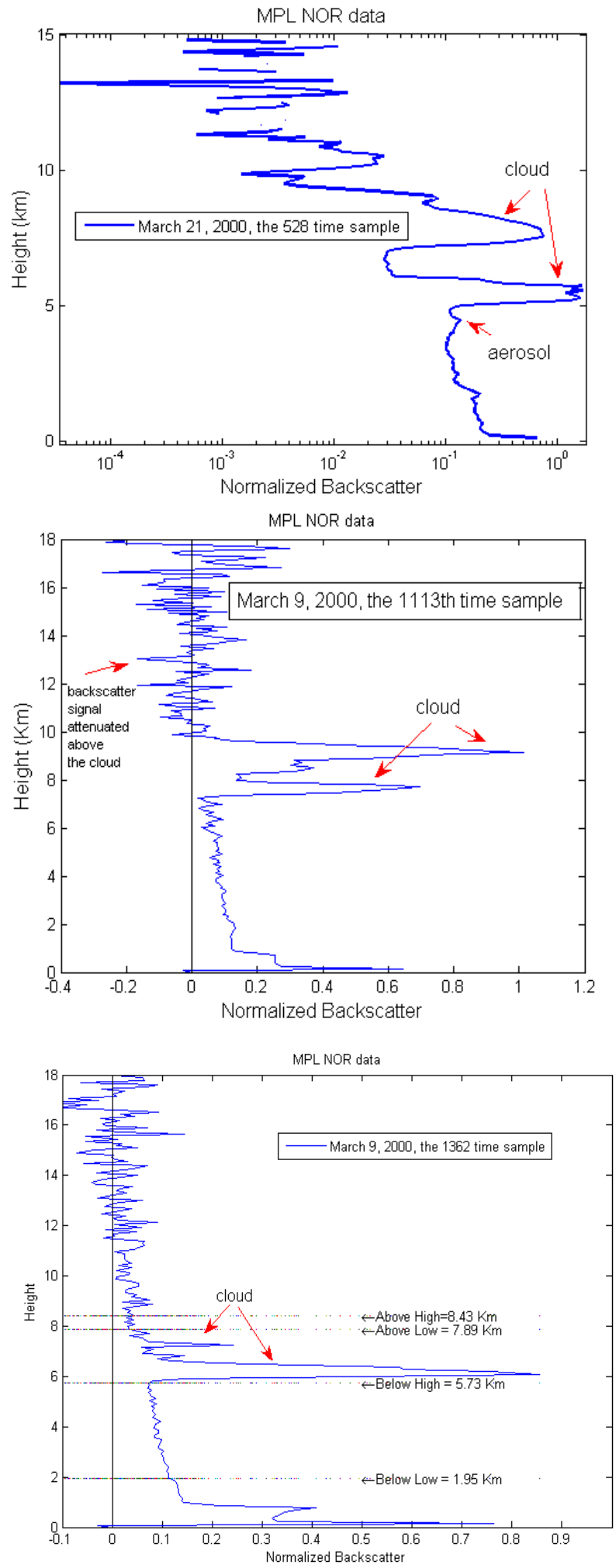

Figure 1. Top panel: Example of the aerosol contamination below the cloud. Middle panel: Example of the normalized backscatter profile attenuated above the cloud. Bottom panel: Example of the successful determination of the cloud boundaries. 
lidar backscatter signal was not attenuation limited above the cloud, but possible aerosol or virga contamination was detected below the cloud, then we use 5 points below the MPLNOR cloud base to retrieve the optical depth, and the data are flagged based on the failed quality checks.

\section{Optical Depth Retrieval Algorithm}

The visible optical depth $(\tau)$ is computed from the cloud backscatter coefficient $\left(\beta_{c}\right)$ :

$$
\beta_{c}=\frac{G\left(z_{o}, z\right)}{1-\frac{2 \eta}{k} \int_{z_{o}}^{z} G\left(Z_{o}, z^{\prime}\right) d z^{\prime}}-\beta_{m}(z)
$$

and

$$
G\left(z_{o}, z\right)=\beta_{m}\left(z_{o}\right) \frac{S(z) z^{2}}{S\left(z_{o}\right) z_{o}} \times \exp \left[2\left(\frac{8 \pi}{3}-\frac{\eta}{k}\right) \int \beta_{m}\left(z^{\prime \prime}\right) d z^{\prime \prime}\right]
$$

where:

$$
\begin{array}{ll}
\beta_{\mathrm{m}}= & \text { molecular backscatter coefficient (calculated from atmospheric pressure } \\
& \text { and temperature profiles; see Section } 4) \\
\eta= & \text { multiple scattering coefficient (assumed a constant of } 0.8 \text { for these data) } \\
k= & \text { backscatter-to-extinction ratio } \\
\mathrm{z}= & \text { height or range } \\
\mathrm{Z}_{\mathrm{O}}= & \text { boundary height below cloud that is aerosol and cloud free } \\
\mathrm{S}(\mathrm{z})= & \text { normalized lidar backscatter signal }
\end{array}
$$

The optical depth is then

$$
\tau=\frac{1}{k} \int_{z_{b}}^{z_{t}} \beta_{c}(z) d z
$$

The integral is performed between cloud base $\left(\mathrm{z}_{\mathrm{b}}\right)$ and cloud top $\left(\mathrm{z}_{\mathrm{t}}\right)$. The parameter $k$ is determined using an iterative technique where $k$ is varied between 0.01 and $0.2 \mathrm{sr}^{-1}$ until the average backscatter coefficient above the cloud is equivalent to the average molecular backscatter coefficient above cloud. For a detailed algorithm description, see Comstock and Sassen (2001). This algorithm has been applied to several long-term datasets in both midlatitudes and the tropics (Sassen and Comstock 2001; Comstock et al. 2002). The optical depth retrieval has also been compared with radar based results (Comstock et al. 2002). 
The Comstock and Sassen (2001) algorithm performs best for upper tropospheric ice clouds. Therefore, we use a slightly different approach for lower level clouds. The lower level clouds are defined when both the lowest cloud base height and highest cloud top height are lower than $5 \mathrm{~km}$. This approach first estimates the cloud optical depth using Beer's Law, such that the two-way transmittance is

$$
T^{2}(z)=\exp \left[-2 \int_{0}^{z} \alpha(z) d z\right]
$$

(e.g., Fernald et al. 1972), where $\alpha$ is the extinction coefficient. The two-way transmittance is computed directly from the lidar backscatter signal such that

$$
T^{2}=\frac{\left(\frac{\bar{\beta}_{a c}}{\bar{\beta}_{m, a c}}\right)}{\left(\frac{\bar{\beta}_{b c}}{\bar{\beta}_{m, b c}}\right)}
$$

where the subscripts "bc" and "ac" denote "below cloud" and "above cloud" respectively. The determination of the below and above cloud free boundaries is described in Section 4. The optical depth is then determined by

$$
\tau=-\left[\ln \left(T^{2}\right) / 2\right]
$$

The $1 / 2$ in Eq. (6) is due to the fact that the lidar signal travels out and back. Eqs. (2) and (3) are then iterated by varying $k$ in Eq. (2) until $\tau$ from Eq. (3) matches the $\tau$ from Eq. (6). This final value of $k$ is passed to the output file.

\section{Quality Control Flags}

The Quality Control (QC) flags and the decisions on turning on/off optical depth (OD) calculations are listed below.

flag_bit_1: No cloud detected - set OD to -9999.0.

flag_bit_2: Possible aerosol or virga contamination below cloud - calculate OD. flag_bit_3: Weak molecular signal above cloud - calculate OD.

flag_bit_4: Cloud base < 200 meters from ground - set OD to -9999.0.

flag_bit_5: No clear air below cloud - set OD to -9999.0.

flag_bit_6: No molecular signal above cloud - set OD to -9999.0.

flag_bit_7: Negative average backscatter below cloud - set OD to -9999.0. flag_bit_8: Negative Rayleigh profile below cloud - set OD to -9999.0.

flag_bit_9: Average backscatter above cloud less than 0.005 - set OD to -9999.0. 
flag_bit_10: Negative Rayleigh profile above cloud - set OD to -9999.0.

flag_bit_11: Negative optical depth retrieved - OD from MPL Variable k is calculated but not from Beer's Law.

\section{Example}

We have applied the algorithm to retrieve the optical depth and its maximum and minimum uncertainties for the thin clouds at the Southern Great Plains (SGP) Central Facility (C1) site for March 2000. The retrieval will be extended to run on other sites/ facilities such as the North Slope of Alaska and Tropical Western Pacific. A quicklook figure is generated for each day, it includes cloud visible optical depth, backscatter-toextinction ratio, cloud boundaries, above and below cloud range bins used to retrieve optical depth, and QC flags for retrieved quantities. Figure 2 shows an example quicklook from March 26, 2000.
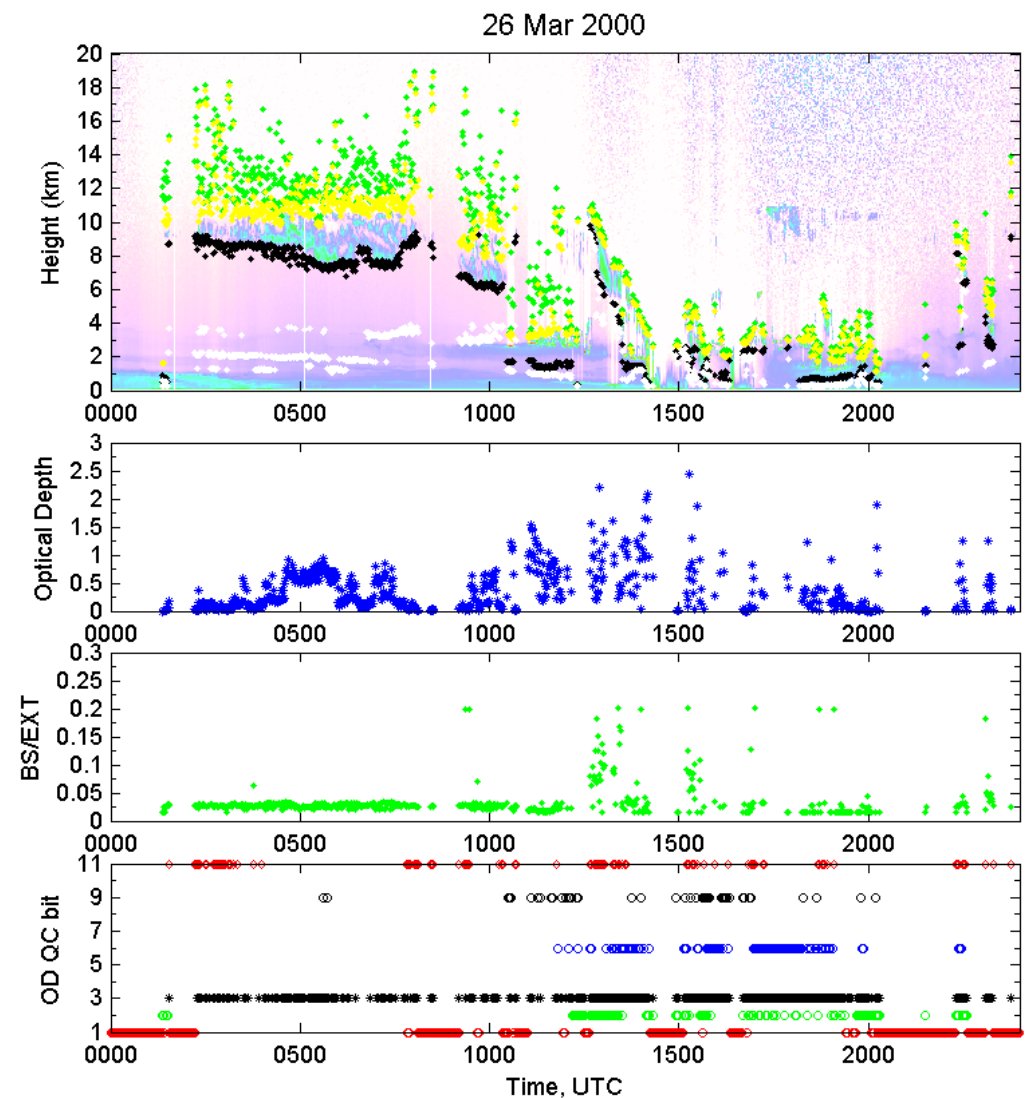

red o: bit 1,No cloud detected.

green o: bit 2,Possible aerosol or virga contamination below cloud. red *: bit 7, Negative average backscatter below cloud. black*: bit 3, Weak molecular signal above cloud. blue *: bit 4, Cloud base $<200$ meters from ground. black o: bit 9 ,Negative average backscatter above cloud blue 0 : bit 6, No molecular signal above cloud. red diamond: bit 11, Negative optical depth retrieved.

Figure 2. From top down: (1) Lidar backscatter intensities and calculated cloud boundaries (green: above-cloud high bin, yellow: above-cloud low bin, black: belowcloud high bin, white: below-cloud low bin) (2) Retrieved optical depth (3) Backscatterto-extinction ratio in $\mathrm{sr}^{-1}$ (BS/EXT) (4) QC bits. 


\section{Conclusions}

The cloud optical depth produced by the MPLCOD VAP is the total column optical, including multi-layer clouds. This VAP does not attempt to provide optical depth for each single layer cloud. The MPL variable "backscatter to extinction ratio" algorithm from Comstock and Sassen (2001) was adapted and the lower clouds optical depth was improved by comparing with that obtained from the Beer's Law. The cloud free regions were carefully examined to assure the accurate optical depth retrievals.

\section{References}

Comstock, JM, and K Sassen. 2001. "Retrieval of cirrus cloud radiative and backscattering properties using combined Lidar and Infrared Radiometer (LIRAD) Measurements.” Journal of Atmospheric and Oceanic Technology, 18:1658-1673.

Comstock, JM, TP Ackerman, and GG Mace. 2002. “Ground-based lidar and radar remote sensing of tropical cirrus clouds at Nauru Island: Cloud statistics and radiative impacts.” Journal of Geophysical Research, 107(23)4714.

Fernald, FG, BM Herman, and JA Reagan. 1972. "Determination of aerosol height distributions by lidar.” Journal of Applied Metrology, 11:482-489.

Measures, RM. 1992. Laser Remote Sensing: Fundamentals and Applications, Krieger Publishing Company, 524 pp.

Sassen, K, and JM Comstock. 2001. "A midlatitude cirrus cloud climatology from the facility for Atmospheric Remote Sensing. Part III: Radiative properties.” Journal of Atmospheric Science, 58:2113-2127. 


\section{Appendix A - Input Data}

Table 1 lists the various ARM data streams used in the MPLCOD VAP for data, along with the specific variables in files that are used in processing.

Table 1. Input Variables

\begin{tabular}{|c|c|c|c|}
\hline Datastream & Variable Name & Variable Long Name & Units \\
\hline \multirow{6}{*}{ sgpmplnor1campC1.c1 } & height & $\begin{array}{l}\text { Vertical height above ground level of } \\
\text { the center of corresponding range_bin }\end{array}$ & km AGL \\
\hline & backscatter & $\begin{array}{l}\text { Normalized corrected relative } \\
\text { backscatter }\end{array}$ & $\begin{array}{c}\text { phe counts * } \\
\mathrm{km}^{\wedge} 2 /(\mathrm{us} * \mathrm{~mJ})\end{array}$ \\
\hline & cloud_mask_2 & Cloud mask & unitless \\
\hline & random_error & $\begin{array}{l}\text { Relative random error in backscatter } \\
\text { profiles }\end{array}$ & fraction \\
\hline & cloud_base_height & Cloud base height & km AGL \\
\hline & cloud_top_height & Cloud top height & $\mathrm{km}$ AGL \\
\hline \multirow{3}{*}{ sgpsondewrpnC1.a1 } & alt & Altitude & $\begin{array}{r}\text { meters above } \\
\text { mean sea level }\end{array}$ \\
\hline & tdry & Dry Bulb Temperature & $\mathrm{C}$ \\
\hline & pres & Pressure & $\mathrm{hPa}$ \\
\hline
\end{tabular}




\section{Appendix B - Output Variables}

Table 2 lists the detail description of the variables for the MPLCOD1COMFLY VAP output file.

Table 1. Output Variables

\begin{tabular}{|c|c|c|}
\hline Fieldname & Description & Unites \\
\hline base_time & Base time in Epoch & $\begin{array}{l}\text { seconds } \\
\text { since } \\
\text { 1970/01/01 } \\
\text { 00:00:00 }\end{array}$ \\
\hline time_offset & Time offset from base_time & $\begin{array}{l}\text { seconds } \\
\text { since } \\
\text { base_time }\end{array}$ \\
\hline time & Time offset from midnight & $\begin{array}{l}\text { seconds } \\
\text { since } \\
\text { midnight }\end{array}$ \\
\hline qc_time & Results of quality checks on sample time & unitless \\
\hline cloud_base_height & Cloud base height & km(AGL) \\
\hline qc_cloud_base_height & Data quality check for cloud base height & unitless \\
\hline cloud_top_height & Cloud top height & $\mathrm{km}(\mathrm{AGL})$ \\
\hline qC_cloud_top_height & Data quality check for cloud top height & unitless \\
\hline below_cloud_lo_bin & $\begin{array}{l}\text { Lowest bin used below cloud base for optical depth } \\
\text { retrieval }\end{array}$ & $\mathrm{km}$ \\
\hline qc_below_cloud_lo_bin & Data quality check for cloud boundaries & unitless \\
\hline below_cloud_hi_bin & $\begin{array}{l}\text { Highest bin used below cloud base for optical depth } \\
\text { retrieval }\end{array}$ & $\mathrm{km}$ \\
\hline qc_below_cloud_hi_bin & Data quality check for cloud boundaries & unitless \\
\hline above_cloud_lo_bin & $\begin{array}{l}\text { Lowest bin used above cloud top for optical depth } \\
\text { retrieval }\end{array}$ & $\mathrm{km}$ \\
\hline qc_above_cloud_lo_bin & Data quality check for cloud boundaries & unitless \\
\hline above_cloud_hi_bin & $\begin{array}{l}\text { Highest bin used above cloud top for optical depth } \\
\text { retrieval }\end{array}$ & $\mathrm{km}$ \\
\hline qc_above_cloud_hi_bin & Data quality check for cloud boundaries & unitless \\
\hline cloud_OD & Total cloud visible optical depth (532 nm) & unitless \\
\hline qC_cloud_OD & Data quality check for cloud_OD & unitless \\
\hline cloud_OD_min & $\begin{array}{l}\text { Minimum optical depth computed if backscatter-to- } \\
\text { extinction ratio is } 0.01 \text { less than the retrieved value. }\end{array}$ & unitless \\
\hline qC_cloud_OD_min & Data quality check for cloud_OD_min & unitless \\
\hline cloud_OD_max & $\begin{array}{l}\text { Maximum optical depth computed if backscatter-to- } \\
\text { extinction ratio is } 0.01 \text { more than the retrieved value. }\end{array}$ & unitless \\
\hline qc_cloud_OD_max & Data quality check for cloud_OD_max & unitless \\
\hline backscatter_to_extinction_ratio & Backscatter-to-extinction ratio & $1 / \mathrm{sr}$ \\
\hline qc_backscatter_to_extinction_ratio & $\begin{array}{l}\text { Data quality check for } \\
\text { backscatter_to_extinction_ratio }\end{array}$ & unitless \\
\hline
\end{tabular}

